\title{
HOXA1 upregulation is associated with poor prognosis and tumor progression in breast cancer
}

\author{
JINTAO LIU ${ }^{1}$, JINQUAN LIU ${ }^{2}$ and XINYI LU ${ }^{1}$ \\ ${ }^{1}$ Department of Breast Surgery, Dalian Central Hospital Affiliated to Dalian Medical University, Dalian, Liaoning 116033; \\ ${ }^{2}$ Department of Clinical Medicine, Datong University School of Medicine, Datong, Shanxi 037009, P.R. China
}

Received July 12, 2018; Accepted December 6, 2018

DOI: $10.3892 /$ etm.2018.7145

\begin{abstract}
Breast cancer (BC) is the most commonly diagnosed cancer and the second leading cause of cancer-associated mortality among females worldwide. As a member of the homeobox (HOX) gene family, HOXA1 is involved in tumor progression and prognosis in several types of human cancer. However, the clinical significance and biological functions of HOXA1 in BC remains unknown. The current study assessed the expression of HOXA1 in BC tissues and cells via western blotting and reverse transcription-quantitative polymerase chain reaction. The association between HOXA1 expression and the clinicopathological features of patients with $\mathrm{BC}$ was analyzed using the Chi-square test. The overall survival of patients was calculated using the Kaplan-Meier method and examined using the log-rank test. Cell proliferation was examined via an MTT assay. Cell cycle distribution and cell apoptosis were analyzed using flow cytometry. The current study demonstrated that HOXA1 mRNA and protein expression was upregulated in BC. In addition, HOXA1 overexpression was associated with poor prognosis and advanced clinicopathological features in patients with $\mathrm{BC}$. Furthermore, knockdown of HOXA1 significantly inhibited cell proliferation by enhancing cell apoptosis and cell cycle arrest in BC cells, which was accompanied with aberrant expression of cell cycle and apoptosis-associated proteins, cyclin D1, B-cell lymphoma 2 (Bcl-2) and Bcl-2-like protein 4. Taken together, the results suggested that HOXA1 may serve as a novel prognostic marker and therapeutic target in BC.
\end{abstract}

\section{Introduction}

Breast cancer (BC) is the most commonly diagnosed cancer and the second leading cause of cancer-associated mortality among women worldwide (1). In 2017, 252,710 new cases of

Correspondence to: Dr Jintao Liu, Department of Breast Surgery, Dalian Central Hospital Affiliated to Dalian Medical University, 826 Southwest Road, Dalian, Liaoning 116033, P.R. China

E-mail: cancer1979@126.com

Key words: homeobox A1, breast cancer, prognosis, progression invasive BC and 40,610 BC-associated mortalities occurred among females in the US (2). In China, an estimated 272,400 new cases and 70,700 BC-associated mortalities were reported in 2015 (3). The current primary treatment strategy for BC is surgical resection followed by hormonal therapy, chemotherapy, radiotherapy and/or biological therapy (4). Although recent advances in early diagnosis and targeted therapies in $\mathrm{BC}$ have been achieved, the molecular mechanism of $\mathrm{BC}$ progression remains poorly understood and the prognosis of patients with advanced $\mathrm{BC}$ remains low. It is therefore important to identify novel biomarkers associated with clinical prognosis that are also involved in the progression of $\mathrm{BC}$.

The homeobox (HOX) gene family encodes homeodomain-containing transcription factors that participate in various physiological processes, which include embryonic development, cell proliferation and differentiation (5). In vertebrates, the HOX gene family contains 39 Hox genes, which are organized into four different chromosome clusters, each containing 9-11 genes $(5,6)$. As a member of the HOX gene family, HOXA1 was previously reported to be involved in tumor progression and prognosis in certain types of human cancer (7-9). Yuan et al (7) reported that elevated HOXA1 expression levels enhance cell proliferation and poor prognosis in gastric cancer. In addition, Wang et al (8) demonstrated that HOXA1 is upregulated in prostate cancer and knockdown of HOXA1 suppresses the growth, invasion and migration of prostate cancer cells. Furthermore, Zha et al (9) reported that HOXA1 overexpression correlates with poor prognosis in patients with hepatocellular carcinoma. However, whether HOXA1 is associated with the prognosis of patients with $\mathrm{BC}$ remains unknown.

In the current study, the expression of HOXA1 in BC tissue and adjacent non-cancerous tissue samples from patients with $\mathrm{BC}$ was examined. The prognosis of patients with $\mathrm{BC}$ with high and low HOXA1 mRNA expression levels was assessed. In addition, the association between HOXA1 expression and clinicopathological features of patients with BC was examined. Furthermore, the effect of HOXA1 expression on $\mathrm{BC}$ cell proliferation, apoptosis and cell cycle distribution was assessed. The present study demonstrated that HOXA1 was upregulated in BC. HOXA1 overexpression was associated with a poor prognosis and advanced clinicopathological features in patients with BC. In addition, 
knockdown of HOXA1 significantly inhibited cell proliferation by enhancing cell apoptosis and cell cycle arrest in BC cells, which was accompanied with the aberrant expression of cell cycle and apoptosis-associated proteins, cyclin D1, B-cell lymphoma 2 (Bcl-2) and Bcl-2-like protein 4. The current study demonstrated that HOXA1 may act as a novel marker in $\mathrm{BC}$ prognosis and a potential therapeutic target for the treatment of BC.

\section{Materials and methods}

Tissue collection. BC tissue and adjacent non-cancerous tissue samples were collected from 45 female patients (age range, 40-72 years) who underwent surgical resection between January 2007 and December 2012 at Dalian Central Hospital Affiliated to Dalian Medical University (Dalian, China). Following surgery, fresh tissue samples were frozen in liquid nitrogen and stored at $-80^{\circ} \mathrm{C}$ until further use. All samples were evaluated and subject to histological diagnosis according to the World Health Organization criteria by two pathologists (10). All patients had regular follow-ups (1-60 months; median follow-up time, 52.4 months) following surgical resection and overall survival was recorded. The clinicopathological features of each patient, including age, tumor size, tumor, node and metastasis (TNM) stage and lymph node metastasis, as well as biological parameters, including estrogen receptor (ER), progesterone receptor (PR) and human epidermal growth factor receptor (HER)-2 status were recorded (Table I). The current study was conducted according to the World Medical Association Declaration of Helsinki and approved by the Ethics Committee of Dalian Central Hospital Affiliated to Dalian Medical University. Written informed consent was obtained from each patient prior to surgery.

Cell culture. Human BC cell lines BT549, T47D,MDA-MB-231 and MCF7 as well as the human breast epithelial cell line MCF-10A were obtained from the Cell Culture Collection of the Chinese Academy of Sciences (Shanghai, China). The four BC cell lines were cultured in Dulbecco's modified Eagle's medium (DMEM) supplemented with $10 \%$ fetal bovine serum (FBS) and $100 \mathrm{U} / \mathrm{ml}$ penicillin/streptomycin mixture (all Gibco; Thermo Fisher Scientific, Inc., Waltham, MA, USA). MCF-10A cells were cultured in RPMI-1640 medium supplemented with $10 \% \mathrm{FBS}$ and $100 \mathrm{U} / \mathrm{ml}$ penicillin/streptomycin mixture (both Gibco; Thermo Fisher Scientific, Inc.). Cells were maintained at $37^{\circ} \mathrm{C}$ in a humidified incubator with $5 \% \mathrm{CO}_{2}$.

Cell transfection. Cells were seeded into six-well plates at $30-40 \%$ confluence and following a $12 \mathrm{~h}$ incubation, cells were transfected with small interfering RNA (siRNA) targeting HOXA1 (si-HOXA1) or scrambled negative control siRNA (si-NC) using Lipofectamine ${ }^{\circledR} 2000$ (Invitrogen; Thermo Fisher Scientific, Inc.), according to the manufacturer's protocol. si-HOXA1 (5'-CAACAAGUACCUUAC ACGA-3') and si-NC (5'-UUCUCCGAACGUGUCACG UTT-3') were purchased from Shanghai GenePharma Co. Ltd. (Shanghai, China). Following 48 h of transfection, cells were harvested and transfection efficiency was detected by
Table I. Association between HOXA1 expression and clinicopathological features of female patients with BC.

\begin{tabular}{|c|c|c|c|c|}
\hline \multirow[b]{2}{*}{$\begin{array}{l}\text { Clinicopathological } \\
\text { features }\end{array}$} & \multirow[b]{2}{*}{$\begin{array}{l}\text { Cases } \\
\text { (n) }\end{array}$} & \multicolumn{2}{|c|}{ HOXA1 expression } & \multirow[b]{2}{*}{ P-value } \\
\hline & & $\begin{array}{l}\text { Low } \\
(n=22)\end{array}$ & $\begin{array}{l}\text { High } \\
(n=23)\end{array}$ & \\
\hline \multicolumn{5}{|l|}{ Age (years) } \\
\hline$\leq 50$ & 18 & 8 & 10 & 0.626 \\
\hline$>50$ & 27 & 14 & 13 & \\
\hline \multicolumn{5}{|l|}{ Tumor size (cm) } \\
\hline$\leq 3$ & 17 & 10 & 7 & 0.299 \\
\hline$>3$ & 28 & 12 & 16 & \\
\hline \multicolumn{5}{|l|}{ ER status } \\
\hline Negative & 20 & 11 & 9 & 0.463 \\
\hline Positive & 25 & 11 & 14 & \\
\hline \multicolumn{5}{|l|}{ PR status } \\
\hline Negative & 19 & 11 & 8 & 0.302 \\
\hline Positive & 26 & 11 & 15 & \\
\hline \multicolumn{5}{|l|}{ HER2 status } \\
\hline Negative & 18 & 10 & 8 & 0.465 \\
\hline Positive & 27 & 12 & 15 & \\
\hline \multicolumn{5}{|l|}{ Lymph node status } \\
\hline Negative & 25 & 16 & 9 & $0.023^{\mathrm{a}}$ \\
\hline Positive & 20 & 6 & 14 & \\
\hline \multicolumn{5}{|l|}{ TNM stage } \\
\hline I-II & 26 & 17 & 9 & $0.01^{\mathrm{a}}$ \\
\hline III & 19 & 5 & 14 & \\
\hline
\end{tabular}

${ }^{\mathrm{a}} \mathrm{P}<0.05$. BC, breast cancer; HOXA1, homeobox A1; ER, estrogen receptor; PR, progesterone receptor; HER2, human epidermal growth factor receptor 2; TNM, tumor, node and metastasis.

reverse transcription-quantitative polymerase chain reaction (RT-qPCR) and western blot analysis prior to functional assays.

$R N A$ extraction and $R T-q P C R$. Total RNA was extracted from tissue samples or cells using TRIzol ${ }^{\circledR}$ reagent (Invitrogen; Thermo Fisher Scientific, Inc.). Total RNA was reverse transcribed into cDNA using a PrimeScript ${ }^{\mathrm{TM}}$ RT-PCR kit (Takara Bio, Inc., Otsu, Japan) for $15 \mathrm{~min}$ at $37^{\circ} \mathrm{C}$ and terminated by heating the samples at $85^{\circ} \mathrm{C}$ for 5 sec. HOXA1 expression was detected by qPCR, which was performed using SYBR-Green PCR Master mix (Applied Biosystems; Thermo Fisher Scientific, Inc.) on the 7300 Real-Time PCR system (Applied Biosystems; Thermo Fisher Scientific, Inc.). The following primer pairs were used for qPCR: HOXA1 forward, 5'-CGGCTTCCTGTG CTAAGTCT-3' and reverse, 5'-TTCATTGTGCCATCCATC AC-3'; and $\beta$-actin forward, 5'-TTAGTTGCGTTACACCCT TTC-3' and reverse, 5'-ACCTTCACCGTTCCAGTTT-3'. The reaction conditions for PCR were as follows: $95^{\circ} \mathrm{C}$ for $3 \mathrm{~min}$ and 40 cycles of $95^{\circ} \mathrm{C}$ for $30 \mathrm{sec}$ and $60^{\circ} \mathrm{C}$ for $30 \mathrm{sec}$. HOXA1 mRNA levels were quantified using the $2^{-\Delta \Delta \mathrm{Cq}}$ 
method (11) and normalized to the internal control $\beta$-actin. RT-qPCR was repeated three times.

Western blot analysis. Total protein was extracted from tissue samples or cells using radioimmunoprecipitation assay buffer (Pierce; Thermo Fisher Scientific, Inc.) supplemented with protease inhibitor (78438; 1:100; Thermo Fisher Scientific, Inc.). Total protein was quantified using a bicinchoninic acid assay and equal amount of protein (30 $\mu \mathrm{g}$ per lane) was separated via SDS-PAGE on a $10 \%$ gel. Separated proteins were transferred to polyvinylidene fluoride membranes and blocked with 5\% non-fat milk at room temperature for $1 \mathrm{~h}$. Membranes were washed three times and incubated with primary antibodies against HOXA1 (ab230513; 1:1,000), $\beta$-actin (ab8227; 1:2,000), cyclin D1 (ab40754; 1:2,000; all Abcam, Cambridge, UK), B-cell lymphoma 2 (cat. no. 4223, 1:1,000) and Bcl-2-like protein 4 (Bax; cat. no. 2774, 1:1,000; both Cell Signaling Technology, Inc., Danvers, MA, USA) overnight at $4^{\circ} \mathrm{C}$. Following primary incubation, membranes were incubated with horseradish peroxidase-conjugated secondary antibodies [horseradish peroxidase (HRP) conjugated goat anti-mouse immunoglobulin G (IgG); sc-2005 and goat anti-rabbit IgG-HRP; sc-2004; 1:5,000; Santa Cruz Biotechnology, Santa Cruz, CA, USA] for $2 \mathrm{~h}$ at room temperature. Protein bands were visualized using the enhanced chemiluminescence detection system (Pierce; Thermo Fisher Scientific, Inc.) with an ECL kit (EMD Millipore, Billerica, MA, USA). Protein expression was quantified using $\beta$-actin as an internal control. Relative protein expression was analyzed using ImageJ software 1.4 (National Institutes of Health, Bethesda, MD, USA). Each experiment was performed in triplicate.

Cell proliferation. To examine the effect of HOXA1 on $\mathrm{BC}$ cell proliferation, MDA-MB-231 and MCF7 BC cell lines were transfected with si-HOXA1 and cell proliferation was assessed using the MTT assay. Briefly, transfected cells were seeded into 96 -well plates at $3 \times 10^{3}$ cells/well and cultured at $37^{\circ} \mathrm{C}$. Following incubation for 24, 48, 72 and $96 \mathrm{~h}, 100 \mu \mathrm{l}$ full DMEM medium containing $0.5 \mathrm{mg} / \mathrm{ml}$ MTT (Sigma-Aldrich; Merck $\mathrm{KGaA}$, Darmstadt, Germany) was added into each well and incubated for a further $4 \mathrm{~h}$ at $37^{\circ} \mathrm{C}$. Subsequently, the medium was removed and $150 \mu \mathrm{l}$ dimethyl sulfoxide (Sigma-Aldrich; Merck KGaA) was added to dissolve the formazan crystals. The absorbance was measured at $490 \mathrm{~nm}$ using a microplate reader (Molecular Devices, USA). Each experiment was performed in triplicate.

Cell cycle analysis. Cell cycle distribution was determined by propidium iodide (PI) staining. Following $48 \mathrm{~h}$ transfection with si-NC or si-HOXA1, MDA-MB-231 and MCF7 cells were seeded into $6-\mathrm{cm}$ dishes at a confluence of $30 \%$ and incubated for $48 \mathrm{~h}$. Cells were subsequently collected and fixed with $70 \%$ ethanol overnight at $4{ }^{\circ} \mathrm{C}$. Cells were washed twice with cold PBS and stained with PI (Sigma-Aldrich; Merck $\mathrm{KGaA}$ ) for $30 \mathrm{~min}$ at room temperature in the dark. The proportion of cells in each phase was analyzed using a BD FACS Calibur Flow Cytometer (BD Biosciences, San Jose, CA, USA) and the data were analyzed using SPSS software (version 18.0; SPSS, Inc., Chicago, IL, USA). Each experiment was performed in triplicate.
Apoptosis assay. Cell apoptosis was analyzed by using the Annexin V-fluorescein isothiocyanate (FITC) Apoptosis Detection kit (BD Pharmingen; BD Biosciences, San Jose, CA, USA). Following $48 \mathrm{~h}$ transfection with si-NC or si-HOXA1, cells were collected via centrifugation at a speed of $50 \mathrm{x} \mathrm{g}$ for $5 \mathrm{~min}$ at room temperature and resuspended in $200 \mu \mathrm{l}$ binding buffer. Cells were subsequently stained with $5 \mu \mathrm{l}$ Annexin V-FITC and $5 \mu \mathrm{l}$ PI together for $15 \mathrm{~min}$ at room temperature in the dark. Apoptotic cells were detected using a BD FACSCalibur Flow cytometer (BD Biosciences) and the rate of apoptosis was analyzed using SPSS software (version 18.0; SPSS, Inc.). Each experiment was performed in triplicate.

Statistical analysis. Data are presented as the mean \pm standard deviation of three replicates. Statistical analyses were performed using SPSS software (version 18.0; SPSS, Inc., Chicago, IL, USA) and GraphPad Prism software (version 6.0; GraphPad Software, La Jolla, CA, USA). Expression levels of HOXA1 mRNA in BC tissue and adjacent non-cancerous tissue samples were compared using the Wilcoxon signed-rank nonparametric test. Student's t-test was used to analyze differences between two groups. Tukey's analysis following one-way analysis of variance was used to analyze differences among multiple groups. The association between HOXA1 expression and clinicopathological features of patients with $\mathrm{BC}$ was analyzed using the Chi-square test. The overall survival of patients was calculated using the Kaplan-Meier method, and the differences between survival curves were examined using the log-rank test. $\mathrm{P}<0.05$ was considered to indicate a statistically significant difference.

\section{Results}

HOXA1 upregulation is associated with poor prognosis in patients with $B C$. To investigate the expression of HOXA1 in $\mathrm{BC}$, the relative HOXA1 protein expression in $\mathrm{BC}$ tissue and adjacent non-cancerous tissue samples from patients with $\mathrm{BC}$ was determined by western blot analysis. HOXA1 protein expression was significantly increased in $\mathrm{BC}$ tissue compared with adjacent non-cancerous tissue samples ( $\mathrm{P}<0.05$; Fig. 1A). In addition, the relative HOXA1 mRNA expression levels in $\mathrm{BC}$ tissue and adjacent non-cancerous tissues from patients with BC were determined by RT-qPCR. HOXA1 mRNA expression levels were significantly increased in $\mathrm{BC}$ tissue compared with adjacent non-cancerous tissue samples ( $\mathrm{P}<0.05$; Fig. 1B). Patients with $\mathrm{BC}$ were divided into two groups: High $(\mathrm{n}=23)$ and low $(n=22)$ HOXA1 expression based on the median mRNA expression level of HOXA1 (3.782 relative to $\beta$-actin) detected in $\mathrm{BC}$ tissue samples from patients with $\mathrm{BC}$. To determine the effect of HOXA1 expression level on the survival of patients with $\mathrm{BC}$, survival curves were plotted using the Kaplan-Meier method and the differences between survival curves were examined using the log-rank test. The overall survival of patients in the high HOXA1 expression group was significantly decreased compared with patients with $\mathrm{BC}$ in the low HOXA1 expression group ( $\mathrm{P}=0.0344$; Fig. 1C). These results suggested that HOXA1 was upregulated in patients with BC and HOXA1 upregulation may be associated with poor prognosis. 

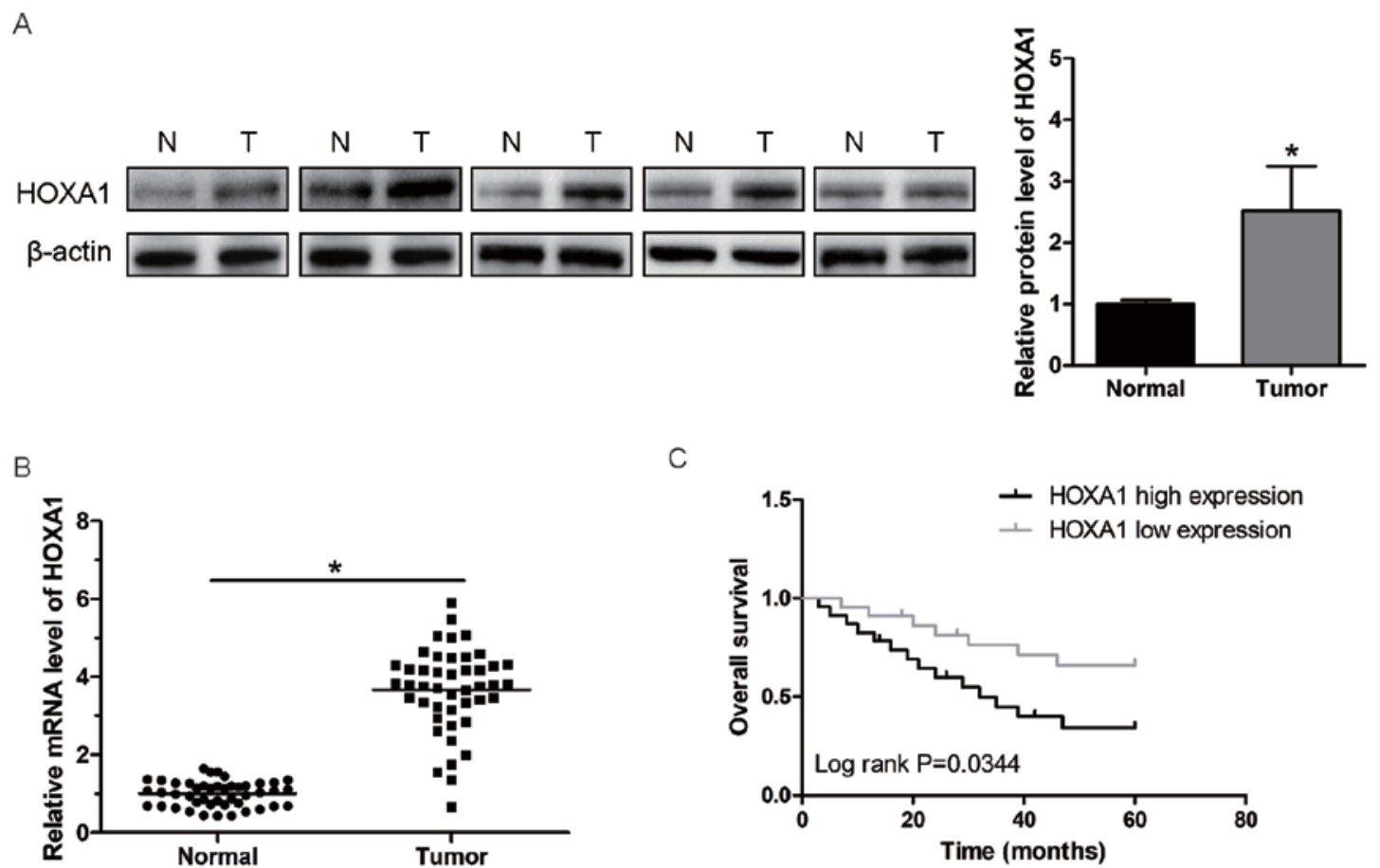

C

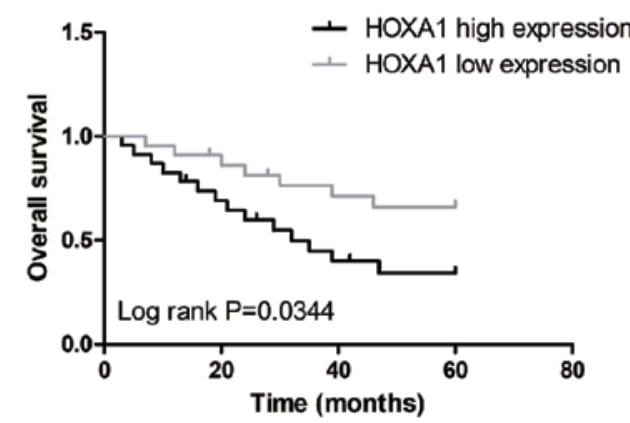

Figure 1. HOXA1 upregulation is associated with poor prognosis in patients with BC. (A) Relative HOXA1 protein expression levels were determined by western blot analysis in BC tissue and adjacent non-cancerous tissue samples from patients with BC. (B) Relative HOXA1 mRNA expression levels were determined by reverse transcription-quantitative polymerase chain reaction in BC tissue and adjacent non-cancerous tissue samples from patients with $\mathrm{BC}$. $\beta$-actin was used as an internal control. (C) Overall survival of patients with BC with high or low HOXA1 expression was analyzed using the Kaplan-Meier method. Data are presented as the mean \pm standard deviation. " $\mathrm{P}<0.05$ vs. Normal. HOXA1, homeobox A1; BC, breast cancer; $\mathrm{T}$, breast cancer tissue; $\mathrm{N}$, adjacent non-cancerous tissue.

HOXA1 upregulation is associated with tumor progression in $B C$. The association between HOXA1 expression and clinicopathological features of patients with $\mathrm{BC}$ was examined (Table I). The mRNA expression level of HOXA1 was significantly associated with lymph node metastasis $(\mathrm{P}=0.023)$ and TNM staging $(\mathrm{P}=0.01)$; however, no association with age, tumor size, and ER, PR or HER2 status was identified $(\mathrm{P}>0.05)$. These results suggested that HOXA1 upregulation may be associated with tumor progression in $\mathrm{BC}$.

HOXA1 siRNA effectively silences HOXA1 gene expression in $B C$ cells. To further confirm HOXA1 overexpression in BC, the mRNA and protein expression levels were determined in BC cell lines BT549, T47D, MDA-MB-231 and MCF7 and the normal breast epithelial cell line MCF-10A. HOXA1 mRNA expression levels were significantly increased in $\mathrm{BC}$ cell lines compared with MCF-10A ( $\mathrm{P}<0.05$; Fig. 2A and B). Similarly, western blot analysis revealed that HOXA1 protein expression levels were increased in $\mathrm{BC}$ cell lines compared with MCF-10A (Fig. 2B). To investigate the functional role of HOXA1 in BC progression, BC cell lines MDA-MB-231 and MCF7 were transfected with si-HOXA1 or si-NC and transfection efficiency was confirmed by RT-qPCR and western blot analysis. The results demonstrated that si-HOXA1 significantly downregulated HOXA1 mRNA $(\mathrm{P}<0.05)$ and markedly reduced protein expression in $\mathrm{BC}$ cell lines compared with the si-NC control (Fig. 2C and D).

Knockdown of HOXAl inhibits BC cell proliferation. MTT assays were performed to investigate the effect of
HOXA1 knockdown on cell proliferation in BC cell lines MDA-MB-231 and MCF7. Knockdown of HOXA1 significantly inhibited BC cell proliferation compared with si-NC at $\geq 72 \mathrm{~h}$ ( $\mathrm{P}<0.05$; Fig. $3 \mathrm{~A}$ and $\mathrm{B})$. In addition, PI staining was used to assess cell cycle distribution using flow cytometry in BC cell lines MDA-MB-231 and MCF7 following transfection with si-HOXA1 or si-NC. The percentage of cells in G1 phase was significantly increased, whilst those in $S$ phase were significantly decreased in $\mathrm{BC}$ cells following HOXA1 knockdown ( $\mathrm{P}<0.05$; Fig. $3 \mathrm{C}$ and $\mathrm{D})$. These results suggested that knockdown of HOXA1 may inhibit BC cell proliferation partly by suppressing cell cycle progression.

Knockdown of HOXA1 promotes BC cell apoptosis. Cell apoptosis was analyzed in BC cell lines MDA-MB-231 and MCF7 following transfection with si-HOXA1 or si-NC. Flow cytometry revealed that knockdown of HOXA1 significantly enhanced $\mathrm{BC}$ cell apoptosis $(\mathrm{P}<0.05$; Fig. 4A and $\mathrm{B})$. In addition, western blot analysis was used to determine the expression level of cell cycle and apoptosis-associated proteins cyclin D1, Bax and Bcl-2 following transfection with si-HOXA1 or si-NC. The protein expression levels of cyclin D1 and Bcl-2 markedly decreased, whilst the levels of Bax markedly increased in BC cells following HOXA1 knockdown (Fig. 4C and D). Taken together, these results suggested that knockdown of HOXA1 may inhibit BC cell proliferation partly by inducing cell apoptosis.

\section{Discussion}

$\mathrm{BC}$ is the most common malignancy among females worldwide and as the incidence of $\mathrm{BC}$ has been increasing over the past 


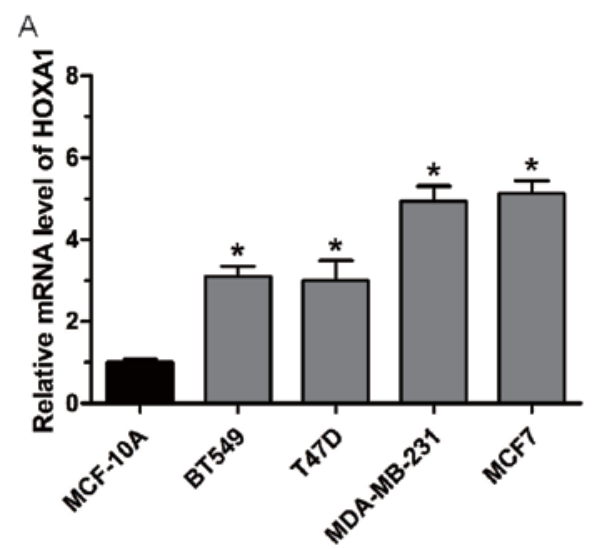

B
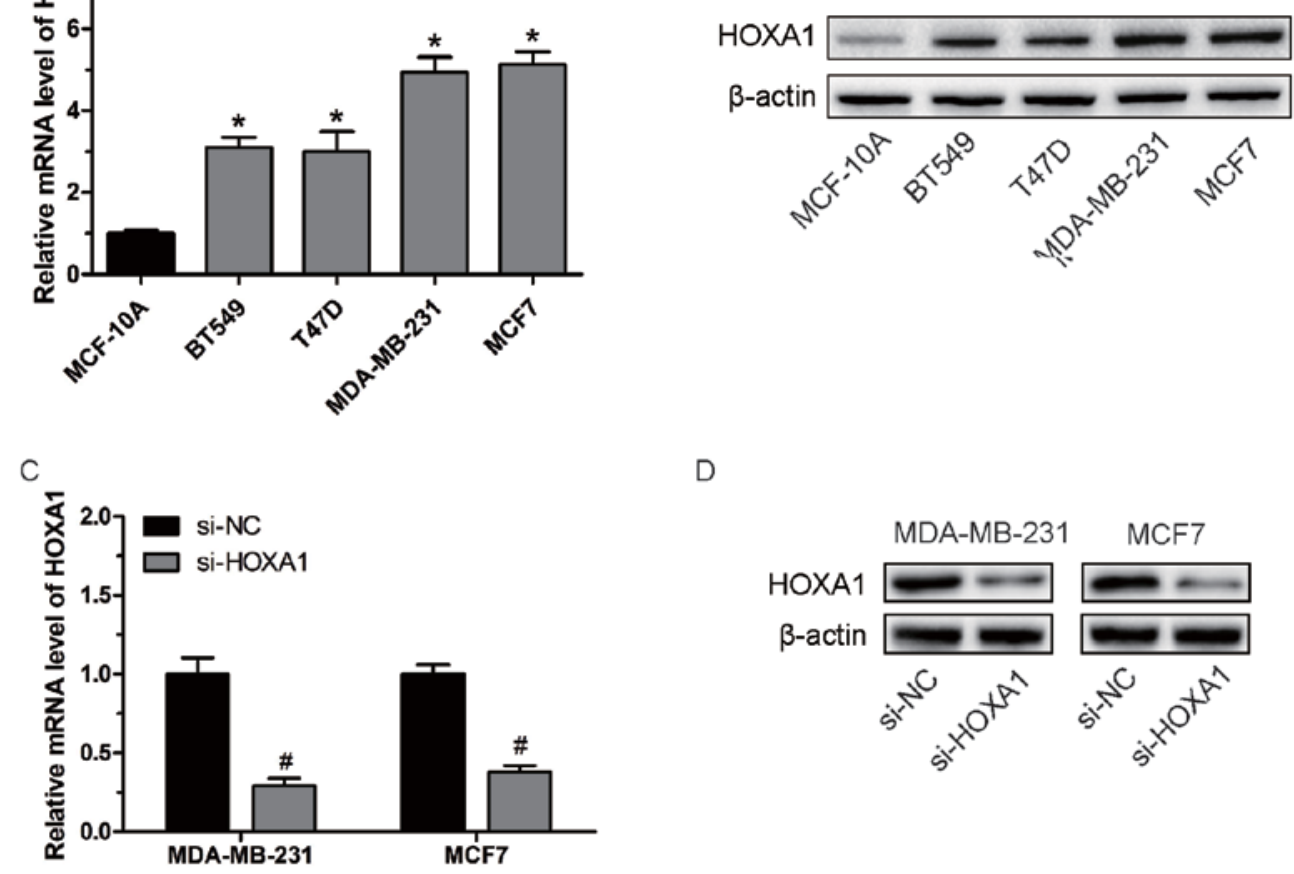

D

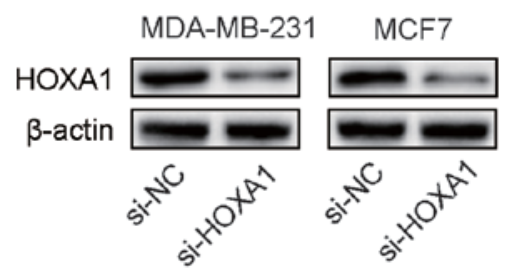

Figure 2. si-HOXA1 effectively silences HOXA1 gene expression in BC cell lines. (A) Relative HOXA1 mRNA expression levels determined by reverse transcription-quantitative polymerase chain reaction in BC cell lines MDA-MB-231, BT549, MCF7 and T47D and the normal breast epithelial cell line MCF-10A. (B) Relative HOXA1 protein expression was determined by western blot analysis in BC cell lines (MDA-MB-231, BT549, MCF7 and T47D) and the normal breast epithelial cell line MCF-10A. (C) Relative HOXA1 mRNA expression level in BC cell lines MDA-MB-231 and MCF7 following transfection with si-HOXA1. (D) Relative HOXA1 protein expression in BC cell lines MDA-MB-231 and MCF7 following transfection with si-HOXA1. Data are presented as the mean \pm standard deviation. " $\mathrm{P}<0.05$ vs. MCF-10A and ${ }^{~} \mathrm{P}<0.05$ vs. si-NC. HOXA1, homeobox A1; BC, breast cancer; si, small interfering RNA; NC, negative; si-HOXA1, siRNA targeting HOXA1.

A

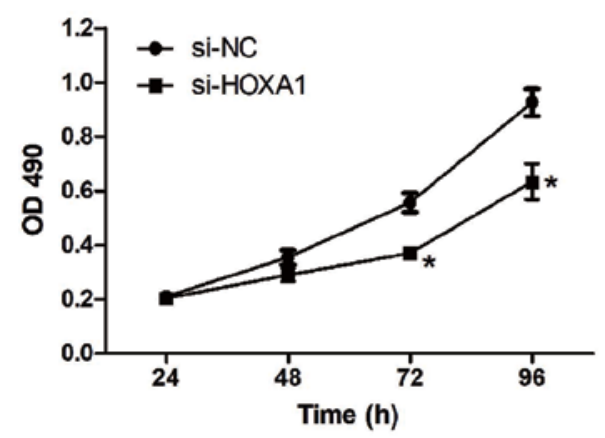

C

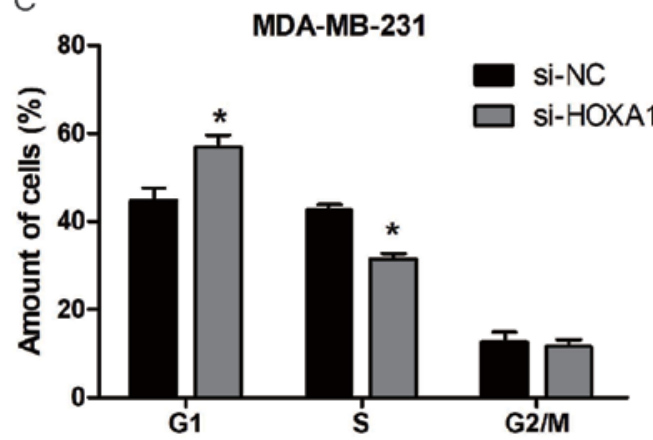

B

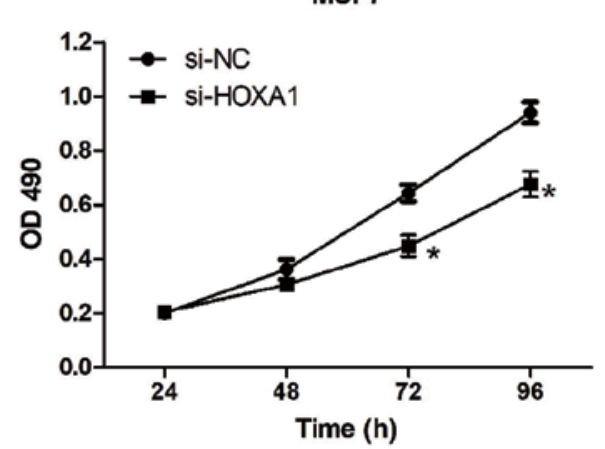

$\mathrm{D}$

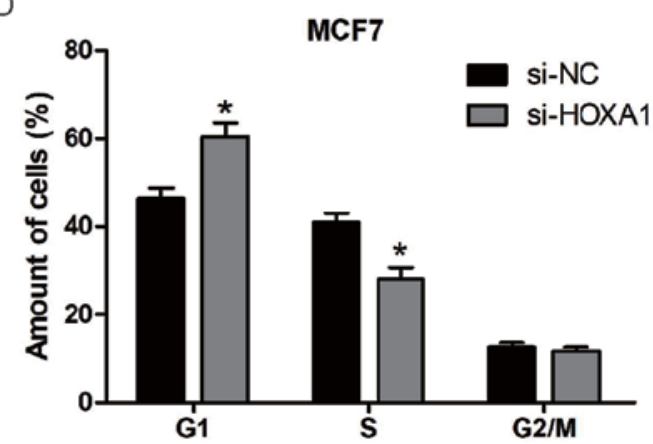

Figure 3. Knockdown of HOXA1 inhibits BC cell proliferation. Cell proliferation was examined in (A) MDA-MB-231 and (B) MCF7 BC cell lines by MTT assay following transfection with si-HOXA1. Cell cycle distribution was examined in (C) MDA-MB-231 and (D) MCF7 BC cell lines by flow cytometry following transfection with si-HOXA1 or si-NC. Data are presented as the mean \pm standard deviation. " $\mathrm{P}<0.05$ vs. si-NC. HOXA1, homeobox A1; BC, breast cancer; si, small interfering RNA; NC, negative; si-HOXA1, siRNA targeting HOXA1. 
A
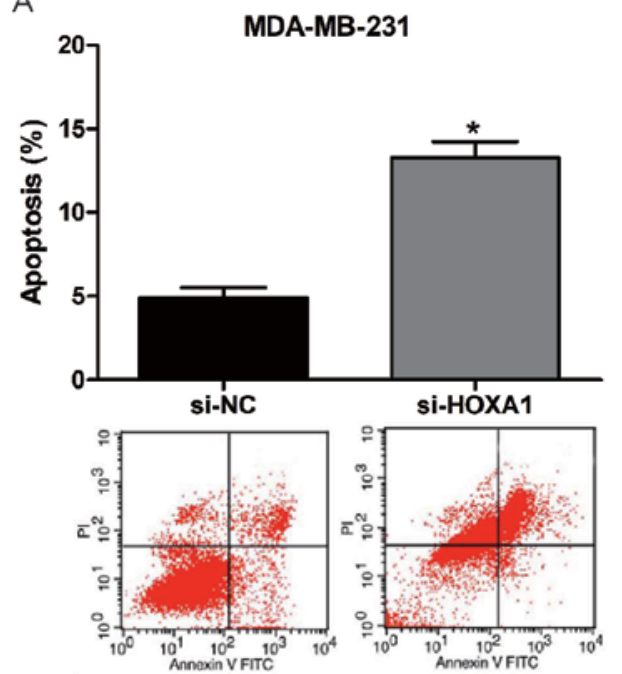

C

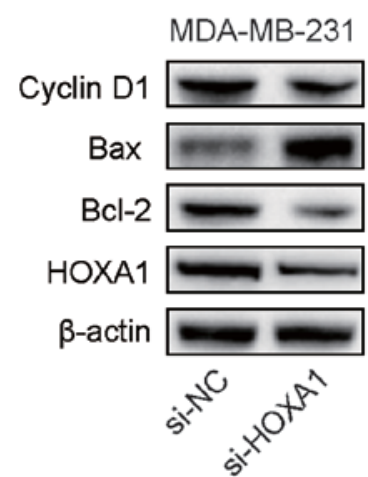

B
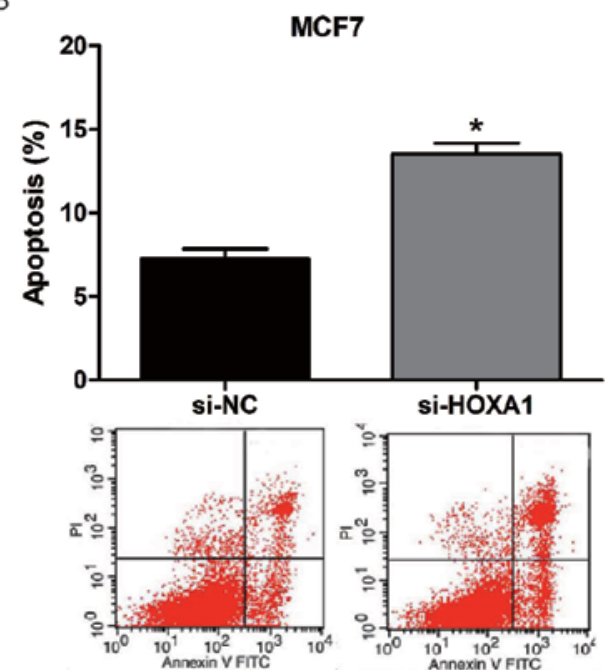

D

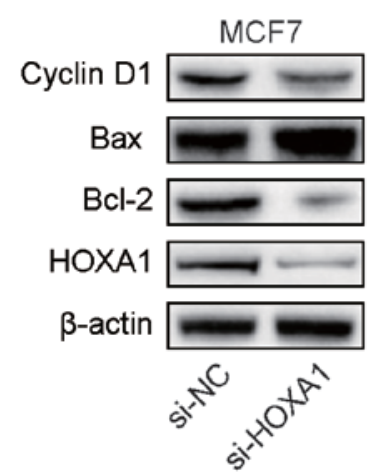

Figure 4. Knockdown of HOXA1 promotes BC cell apoptosis. Cell apoptosis was analyzed in (A) MDA-MB-231 and (B) MCF7 BC cell lines by flow cytometry following transfection with si-HOXA1. Relative protein expression of cyclin D1, Bax and Bcl-2 was determined by western blot analysis in (C) MDA-MB-231 and (D) MCF7 BC cell lines following transfection with si-HOXA1. Data are presented as the mean \pm standard deviation. "P<0.05 vs. si-NC. HOXA1, homeobox A1; BC, breast cancer; si, small interfering RNA; NC, negative; si-HOXA1, siRNA targeting HOXA1; Bcl-2, B-cell lymphoma 2; Bax, Bcl-2-like protein 4; PI, propidium iodide; FITC, fluorescein isothiocyanate.

years, it is considered to be a serious health risk $(12,13)$. Thus, a better understanding of the molecular mechanisms involved in $\mathrm{BC}$ initiation and progression as well as validation of novel prognostic biomarkers may have a potential clinical significance for patients with BC. Previous studies demonstrated that several HOX genes, including HOXA5, HOXB9 and HOXC8 were aberrantly expressed in $\mathrm{BC}$ and served roles in tumor progression in BC (14-16). Additionally, HOXA1 is involved in the development and prognosis of several types of human cancer, including gastric cancer, prostate cancer and hepatocellular carcinoma (7-9). However, the clinical significance of HOXA1 in BC remains unknown.

In the current study, the mRNA and protein expression levels of HOXA1 in BC tissue and adjacent non-cancerous tissue samples were determined via RT-qPCR and western blot analysis, respectively. The results demonstrated that both HOXA1 mRNA and protein expression levels were significantly upregulated in BC tissue compared with non-cancerous tissue samples from patients with BC. Survival analysis indicated that patients with high HOXA1 expression had a significantly poorer overall survival compared with patients with low HOXA1 expression. In addition, the association between HOXA1 expression and clinicopathological features of patients with $\mathrm{BC}$ was examined and HOXA1 expression was associated with lymph node metastasis and TNM staging. No association with age, tumor size and ER, PR or HER2 status was identified. Taken together, these results suggested that HOXA1 upregulation may be associated with poor prognosis and tumor progression in BC.

To investigate the biological function of HOXA1 in $\mathrm{BC}$, BC cell lines MDA-MB-231 and MCF7 were transfected with si-HOXA1 or si-NC. Knockdown of HOXA1 significantly inhibited cell proliferation and induced apoptosis and cell cycle arrest in BC cells. Furthermore, the expression of cell cycle and apoptosis-associated proteins, cyclin D1, Bax and Bcl-2 were examined. Western blot analysis revealed that the protein expression levels of cyclin D1 and Bcl-2 decreased, whilst the level of Bax increased following knockdown of HOXA1 in $\mathrm{BC}$ cells. Together, these results suggest that HOXA1 may be involved in the development of $\mathrm{BC}$.

The underlying mechanism of HOXA1 in BC progression was not investigated in the current study and remains unclear. A recent study demonstrated that HOXA1 activates nuclear factor (NF) $-\kappa \mathrm{B}$ and operates upstream of the $\mathrm{NF}-\kappa \mathrm{B}$ inhibitor, I $\kappa \mathrm{B}$ to modulate the tumor necrosis factor- $\alpha / \mathrm{NF}-\kappa \mathrm{B}$ signaling pathway (17). In addition, a previous study reported 
that HOXA1 regulates tumor progression in gastric cancer via cyclin D1 (7). Furthermore, previous studies demonstrated that in different types of cancer, HOXA1 is regulated by several microRNAs (miR), including miR-99a, miR-30c and miR-100 (18-20). Therefore, further studies are required to investigate the underlying mechanism of HOXA1 in BC.

In conclusion, the current study demonstrated that HOXA1 mRNA and protein expression levels were significantly upregulated in BC. In addition, HOXA1 overexpression was associated with poor prognosis and tumor progression in BC. Furthermore, knockdown of HOXA1 significantly inhibited cell proliferation by inducing apoptosis and cell cycle arrest in BC cells. Together, these findings suggested that HOXA1 may serve as a novel prognostic marker and a potential therapeutic target in $\mathrm{BC}$.

\section{Acknowledgements}

Not applicable.

\section{Funding}

No funding was received.

\section{Availability of data and materials}

The datasets used and/or analyzed during the current study are available from the corresponding author on reasonable request.

\section{Authors' contributions}

JintL designed the current study. JintL and JinqL performed the experiments. JinqL and XL collected and analyzed the data. JintL and XL wrote and revised the manuscript.

\section{Ethics approval and consent to participate}

The current study was approved by the Ethics Committee of Dalian Central Hospital Affiliated to Dalian Medical University. Written informed consent was obtained from each patient.

\section{Patient consent for publication}

Patients provided written informed consent for the publication of any associated data from their samples.

\section{Competing interests}

The authors declare that they have no competing interests.

\section{References}

1. Siegel RL, Miller KD and Jemal A: Cancer statistics, 2018. CA Cancer J Clin 68: 7-30, 2018
2. DeSantis CE, Ma J, Goding Sauer A, Newman LA and Jemal A: Breast cancer statistics, 2017, racial disparity in mortality by state. CA Cancer J Clin 67: 439-448, 2017.

3. Chen W, Zheng R, Baade PD, Zhang S, Zeng H, Bray F, Jemal A Yu XQ and He J: Cancer statistics in China, 2015. CA Cancer J Clin 66: 115-132, 2016.

4. Qin C, Zhao Y, Gong C and Yang Z: MicroRNA-154/ADAM9 axis inhibits the proliferation, migration and invasion of breast cancer cells. Oncol Lett 14: 6969-6975, 2017.

5. Gehring WJ and Hiromi Y: Homeotic genes and the homeobox. Annu Rev Genet 20: 147-173, 1986.

6. Scott MP: Vertebrate homeobox gene nomenclature. Cell 71: 551-553, 1992.

7. Yuan C, Zhu X, Han Y, Song C, Liu C, Lu S, Zhang M, Yu F, Peng Z and Zhou C: Elevated HOXA1 expression correlates with accelerated tumor cell proliferation and poor prognosis in gastric cancer partly via cyclin D1. J Exp Clin Cancer Res 35: $15,2016$.

8. Wang H, Liu G, Shen D, Ye H, Huang J, Jiao L and Sun Y: HOXA1 enhances the cell proliferation, invasion and metastasis of prostate cancer cells. Oncol Rep 34: 1203-1210, 2015.

9. Zha TZ, Hu BS, Yu HF, Tan YF, Zhang Y and Zhang K: Overexpression of HOXA1 correlates with poor prognosis in patients with hepatocellular carcinoma. Tumour Biol 33: 2125-2134, 2012.

10. Leong AS and Zhuang Z: The changing role of pathology in breast cancer diagnosis and treatment. Pathobiology 78: 99-114, 2011.

11. Livak KJ and Schmittgen TD: Analysis of relative gene expression data using real-time quantitative PCR and the 2(-Delta Delta C(T)) method. Methods 25: 402-408, 2001.

12. Li Y, Lv M, Song Z, Lou Z, Wang R and Zhuang M: Long non-coding RNA NNT-AS1 affects progression of breast cancer through miR-142-3p/ZEB1 axis. Biomed Pharmacother 103: 939-946, 2018.

13. Li P, Dong J, Zhou X, Sun W, Huang H, Chen T, Ye B, Zheng Z and Lu M: Expression patterns of microRNA-329 and its clinical performance in diagnosis and prognosis of breast cancer. Onco Targets Ther 10: 5711-5718, 2017.

14. Teo WW, Merino VF, Cho S, Korangath P, Liang X, Wu RC, Neumann NM, Ewald AJ and Sukumar S: HOXA5 determines cell fate transition and impedes tumor initiation and progression in breast cancer through regulation of E-cadherin and CD24. Oncogene 35: 5539-5551, 2016.

15. Hayashida T, Takahashi F, Chiba N, Brachtel E, Takahashi M, Godin-Heymann N, Gross KW, Vivanco Md, Wijendran V, Shioda T, et al: HOXB9, a gene overexpressed in breast cancer, promotes tumorigenicity and lung metastasis. Proc Natl Acad Sci USA 107: 1100-1105, 2010.

16. Zhang Y, Yang C, Zhang M, Liu H, Gong C, Zhang J, Xu S, Zou J, Kai Y and Li Y: Interleukin enhancer-binding factor 3 and HOXC8 co-activate cadherin 11 transcription to promote breast cancer cells proliferation and migration. Oncotarget 8: 107477-107491, 2017.

17. Taminiau A, Draime A, Tys J, Lambert B, Vandeputte J, Nguyen N, Renard P, Geerts D and Rezsöhazy R: HOXA1 binds RBCK1/HOIL-1 and TRAF2 and modulates the TNF/NF- $\kappa$ B pathway in a transcription-independent manner. Nucleic Acids Res 44: 7331-7349, 2016.

18. Wang JG, Tang WP, Liao MC, Liu YP and Ai XH: MiR-99a suppresses cell invasion and metastasis in nasopharyngeal carcinoma through targeting HOXA1. Onco Targets Ther 10: 753-761, 2017.

19. Ni LY, Zhao JD, Lu YH, Li W, Li BL, Wang XC and Meng QG: MicroRNA-30c suppressed giant-cell tumor of bone cell metastasis and growth via targeting HOXA1. Eur Rev Med Pharmacol Sci 21: 4819-4827, 2017.

20. Xiao F, Bai Y, Chen Z, Li Y, Luo L, Huang J, Yang J, Liao H and Guo L: Downregulation of HOXA1 gene affects small cell lung cancer cell survival and chemoresistance under the regulation of miR-100. Eur J Cancer 50: 1541-1554, 2014. 